\section{Research Square}

Preprints are preliminary reports that have not undergone peer review.

They should not be considered conclusive, used to inform clinical practice, or referenced by the media as validated information.

\title{
Apelin-13 Attenuates Early Brain Injury Through Inhibiting Inflammation and Apoptosis in Rats After Experimental Subarachnoid Hemorrhage
}

\section{Xiaoyan Shen}

First Affiliated Hospital of Soochow University

\section{Guiqiang Yuan}

First Affiliated Hospital of Soochow University

Bing Li

First Affiliated Hospital of Soochow University

Cheng Cao

First Affiliated Hospital of Soochow University

\section{Demao Cao}

First Affiliated Hospital of Soochow University

Jiang Wu

First Affiliated Hospital of Soochow University

\section{Xiang Li}

First Affiliated Hospital of Soochow University Department of General Surgery

\section{Haiying Li}

First Affiliated Hospital of Soochow University

Haitao Shen ( $\nabla$ dagezi120@126.com )

First Affiliated Hospital of Soochow University https://orcid.org/0000-0001-9296-3725

\section{Zhong Wang}

First Affiliated Hospital of Soochow University

\section{Gang Chen}

First Affiliated Hospital of Soochow University

\section{Research Article}

Keywords: Apelin-13, APJ, Inflammation, Apoptosis, Subarachnoid hemorrhage

Posted Date: September 8th, 2021

DOl: https://doi.org/10.21203/rs.3.rs-302819/v1 
License: (c) (i) This work is licensed under a Creative Commons Attribution 4.0 International License. Read Full License

Version of Record: A version of this preprint was published at Molecular Biology Reports on January 9th, 2022. See the published version at https://doi.org/10.1007/s11033-021-07028-y. 


\section{Abstract}

Background: Early brain injury (EBI) has been considered as the major contributor to the neurological dysfunction and poor clinical outcomes of subarachnoid hemorrhage (SAH). Studies showed that apelin13 exhibits a neuroprotective effect in brain damage induced by cerebral ischemia. However, it remains unclear whether apelin-13 could exhibit the protective functions following SAH. The present study aimed to validate the neuroprotective role of apelin-13 in SAH, and further investigated the underlying mechanisms.

Methods and Results: We constructed SAH rat model and we found that apelin-13 significantly alleviated neurological disorder and brain edema, improved memory deficits in SAH rats. Apelin-13 treatment decreased contents of TNF- $\alpha$ and IL-1 $\beta$ in cerebral spinal fluid of SAH rat by using ELISA. Apelin-13 treatment promoted the expression of APJ and Bcl-2, and decreased the level of active caspase- 3 and Bax in the temporal cortex after SAH by using western blot. Also, apelin-13 attenuated the cortical cell death and neuronal degeneration as shown by TUNEL, FJB and Nissl staining. However, ML221, an inhibitor of APJ, significantly reversed all the above neuroprotective effects of apelin-13. Moreover, a neuronmicroglia co-culture system, which mimic SAH in vitro, confirmed the protective effect of apelin-13 on neurons and the inhibitory effect on inflammation through apoptosis-related proteins.

Conclusions: These data demonstrated that apelin-13 exhibit a neuroprotective role after SAH through inhibition of apoptosis in an APJ dependent manner.

\section{Introduction}

Subarachnoid hemorrhage (SAH) is reported to be related with elevated rates of mortality and morbidity, which accounts for about $5 \%$ of all stroke cases [1]. Rupture of a cerebral aneurysm which located at the skull base is the most common cause in $85 \%$ spontaneous SAH cases [2]. Despite significant improvements attributed to the microsurgical aneurysm clipping and endovascular interventional therapy, the outcome of SAH is still dissatisfactory. Effects are limited to overcome brain damage and improve the ending of SAH, especially the poor-grade ones [3]. Following SAH, previous literature has reported the occurrence of early brain injury (EBI) and cognitive deficits. EBI has been regarded as the major contributor to the neurological dysfunction and poor clinical outcomes in patients following SAH $[4,5]$. Thus, therapeutic inhibition of EBI may represent a novel and promising approach in the management of $\mathrm{SAH}$ cases. However, the precise underlying mechanism of the pathogenesis of EBI remains obscure, greatly impeding the development of effective therapeutic modality for EBI [6, 7]. Therefore, elucidating the underlying molecular mechanism of EBI is critical for develop novel and effective therapeutic approach against SAH.

Apelin is a ligand of $\mathrm{G}$ protein-coupled receptor-apelin and angiotensin-1-like receptor (APJ). After translocation, apelin cleavages into four different peptide components, such as apelin-17, apelin-36, apelin-12, and apelin-13. Of note, apelin-13 is one of the most active form of apelin, possessing the 
highest plasma concentration and greatest biological activity [8]. Accumulating evidences demonstrated that apelin-13 functions as a protective factor against brain injury. Apelin-13 could decrease brain water content, inhibit cell apoptosis, and improve motor function in an animal model following intracerebral hemorrhage [9]. Moreover, apelin-13 exhibits neuroprotective roles in brain ischemic/reperfusion injury induced by reactive oxide species (ROS)-mediated oxidative stress and inflammatory response; mechanically, apelin-13 activates AMPK/GSK-3 $\beta$ pathway and further enhances the expression of Nrf2regulated antioxidant enzymes [10]. Taken together, apelin-13 may represent a potential target for therapeutic intervention in brain injury. However, the protective effect of apelin-13/APJ system in SAH remains not fully elucidated.

In this research, we evaluated the effects of apelin-13 on neurological impairment, brain edema, neuroinflammation and spatial memory deficits in a rat SAH model. In addition, apelin-13 was administrated intracerebroventricularly in SAH model of rats and the neuronal apoptosis and degeneration were evaluated. Moreover, an APJ antagonist, ML221 (4-oxo-6-((pyrimidin-2ylthio)methyl)-4H-pyran-3-yl 4-nitrobenzoate), was used to furthermore investigate the neuroprotective efficacy of apelin-13/APJ signaling following SAH. Finally, the neuroprotective effect of apelin-13/APJ signaling was verified in vitro SAH model.

\section{Materials And Methods}

\section{Ethics and Animals}

Animals were purchased from the Animal Center of Soochow University (Suzhou, China). These adult male Sprague-Dawley (SD) rats weighing $280 \mathrm{~g}$ to $320 \mathrm{~g}$ were housed under a 12:12 h light-dark cycle and a temperature of $23 \pm 1{ }^{\circ} \mathrm{C}$ and humidity of $40 \%$. Food and water were provided ad libitum. We used these animals and abided by the guidelines of the National Institutes of Health. Manipulation of animals were approved by the Institutional Animal Care Committee of Soochow University.

\section{Animal Study Design and Drug Administration}

This animal study was divided into two parts: experiment 1 and experiment 2, and the experimental design was shown in Fig. 1. All rats were numbered and divided into several groups using a table of random numbers by a technician who was entirely blind to this study. In behavioral impairment, brain edema, ELISA and Morris water maze (MWM) test, the technician was entirely blind to this experimental groups.

In the experiment 1 (Fig. 1a), a total of 150 SD rats was prepared while 108 rats survived (18 rats come from the Sham group, and 90 rats surviving after surgery from an initial 132 rats in the SAH group). Survival rats were randomly classified into six groups (18 rats per group): Sham group; $\mathrm{SAH} ; \mathrm{SAH}+$

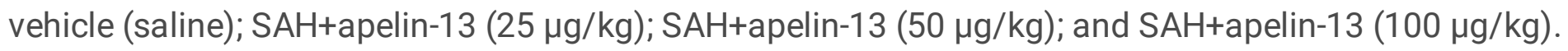


For SAH group: To establish a rat model of SAH [11], animals were anesthetized following intraperitoneal injection of $4 \%$ chloral hydrate at a dosage of $1 \mathrm{~mL} / 100 \mathrm{~g}$. Then, the rat lay prostrate with its head located in a suitable position by fixing firmly in a stereotaxic apparatus (Zhenghua Biologics, Anhui, China). The bregma was exposed though a middle scalp incision. With the site of $7.5 \mathrm{~mm}$ anterior to the bregma, a miniature drilling was used to create a $1 \mathrm{~mm}$ hole. The needle was placed at an angle of $45^{\circ}$ from the sagittal plane, inserted slowly towards the midline. The needle was advanced $11 \mathrm{~mm}$ into the prechiasmatic cistern and reached the depth about 2-3 $\mathrm{mm}$ anterior to the chiasm, and retracted $0.5 \mathrm{~mm}$. Then, $300 \mu \mathrm{L}$ of fresh and non-heparinized blood extracted from left inguen artery were injected into prechiasmatic cistern in $20 \mathrm{~s}$.

For sham group: All the procedures of surgery were same as the SAH group. But, the rat in the sham group underwent administration $300 \mu \mathrm{L}$ saline rather than fresh and non-heparinized blood.

For SAH+apelin-13 group: All the procedures of surgery were same as the SAH group. Rats were administrated apelin-13 immediately after SAH induction. Rats were injected intracerebroventricularly with apelin-13 (ab141010, Abcam, USA) at $25 \mu \mathrm{g} / \mathrm{kg}, 50 \mu \mathrm{g} / \mathrm{kg}$, and $100 \mu \mathrm{g} / \mathrm{kg}$, by using a Hamilton microsyringe. The dosage of apelin-13 was chosen according to the previous article described with minor modifications [12]. The hole for drug injection located lateral $1.5 \mathrm{~mm}$ from the bregma and depth $3.5 \mathrm{~mm}$ from the skull bone. The Hamilton microsyringe was stayed for $5 \mathrm{~min}$ in order to let the drugs dilute into the ventricle maximally.

For SAH+vehicle group: All the procedures were same as the SAH+apelin-13 group. But, the drugs of apelin-13 was replaced by saline.

After surgery, the incision was then sutured and $5 \mathrm{~mL}$ of $0.9 \% \mathrm{NaCl}$ was injected subcutaneously to prevent dehydration of experimental rats. All animals were placed appropriately and recover for $45 \mathrm{~min}$. After recovery, animals were put back to their cages and food and water were provided without restriction. The feeding room was maintained with suitable humidity and was kept at the temperature of $23 \pm 1{ }^{\circ} \mathrm{C}$. The inferior basal temporal lobe was stained with blood if the SAH model was established correctly (Fig. 1b).

In the experiment 2 (Fig. 1C), a total of 152 SD rats was prepared while 120 rats survived (24 rats come from the Sham group, and 96 rats surviving after surgery from an initial 128 rats in the SAH group). Survival rats were randomly classified into five groups (24 rats per group): Sham group; SAH group; SAH+vehicle (DMSO); SAH+apelin-13 (50 $\mathrm{gg} / \mathrm{kg})$; and SAH+apelin-13+ML221.

For Sham group, SAH group, and SAH+apelin-13 (50 $\mu \mathrm{g} / \mathrm{kg})$ group: All surgical procedures were the same as the description above in the experiment 1 . The dosage of $50 \mu \mathrm{g} / \mathrm{kg}$ of apelin-13 was chosen from Experiment 1 according to the results.

For SAH+apelin-13+ML221 group: All surgical procedures were the same as the SAH+apelin-13 (50 $\mu \mathrm{g} / \mathrm{kg}$ ) group, while the difference was that after injecting apelin-13, $50 \mu \mathrm{g} / \mathrm{kg}$ ML221 (SML0919, Sigma, 
USA) [13] was injected immediately in the bone hole.

For $\mathrm{SAH}+$ vehicle group: All surgical procedures were the same as the $\mathrm{SAH}+$ apelin-13 group, while the apelin-13 was replaced by DMSO.

Postoperative management of each group was the same as in experiment 1.

\section{Sample collection}

Animals were sacrificed at the time point of $48 \mathrm{~h}$ post-SAH-modeling except those for MWM test. The MWM experiment began on the second day after SAH modeling and was performed for 4 consecutive days. Neurological score and brain edema were assessed $48 \mathrm{~h}$ after modeling of SAH. For ELISA, the cerebral spinal fluid (CSF) samples were collected by puncturing the cisterna magna, the neck skin was cut open with a straight incision and the muscles were exposed, an injection syringe was slowly inserted towards the cisterna magna, as described previously [14]. Approximately $100 \mu \mathrm{L}$ CSF were extracted and centrifuged immediately and retained supernatant for ELISA detections. The bilateral temporal base brain tissues near to the blood clots (about $100 \mathrm{mg} /$ each rat) (Fig. 1b) were collected for western blotting. The total coronal sections containing temporal base brain tissues (Fig. 1b) were applied to TUNEL, FJB and Nissl staining. In every figure, the "n" represented the sample size.

\section{Cell culture and stimulation}

The primary cortical neurons of rats was purchased from Procell (CP-R105, China) and cultured in complete culture medium of rat cortical neurons (Procell, CM-R105). The primary microglia of BV-2 cells were purchased from Procell (CL-0493, China) and cultured in Minimum Essential Medium (Procell, PM150410), which supplemented 10\% fetal bovine serum and 1\% penicillin-streptomycin. Neuron and BV-2 cells were placed in an incubator at $37^{\circ} \mathrm{C}$ with $5 \% \mathrm{CO}_{2}$. To mimic SAH in vitro (Fig. 1d), neurons and $\mathrm{BV}-2$ cells were induced with $25 \mu \mathrm{M}$ oxyhemoglobin $(\mathrm{OxyHb})$ alone for $24 \mathrm{~h}$ and then transferred to the Transwell co-culture system [15]. The different concentrations of apelin-13 (0.5 nM, $5 \mathrm{nM}, 50 \mathrm{nM})$ was respectively added into co-culture Transwell system and cultured for another $24 \mathrm{~h}$. After co-culture, the morphology of neurons and BV-2 cell were observed and the number was detected by MTT.

According to the results of cell morphology observation and MTT, the optimal concentration of apelin-13 was selected for subsequent experiments. The co-culture system of BV-2 and neurons remained unchanged and was divided into 5 groups: control, SAH, SAH+DMSO, SAH+apelin-13, SAH+apelin13+ML221. For control group, BV-2 and neurons were co-cultured in Transwell system but were not pretreated with $\mathrm{OxyHb}$ exposure. For SAH group, neurons and BV-2 cells were induced with $\mathrm{OxyHb}$ alone for $24 \mathrm{~h}$ and then transferred to the Transwell co-culture system. For SAH+apelin-13 group, after SAH induction, the optimal concentration of apelin-13 was added and the culture continued for $24 \mathrm{~h}$. For SAH+DMSO group, all the procedures were the same as for group SAH+apelin-13, except that apelin-13 was replaced by $10 \% \mathrm{DMSO}$. For SAH+apelin-13+ML221 group, all the procedures were the same as for group SAH+apelin-13, but ML221 was added immediately after apelin-13. After culture, neuronal 
apoptosis was detected by using TUNEL staining, and the expression of apoptosis-related proteins (Bcl2, Bax, and active caspase-3), and inflammatory factors (IL-4, IL-10, TNF-a, and IL-1 $\beta$ ) of BV-2 were detected by using western blotting or immunofluorescence (IF).

\section{Statistical Analysis}

Our data were illustrated as means \pm standard deviation (means \pm SD) and subject to statistical analysis by GraphPad Prism 6.0. The Mann-Whitney U-test for nonparametric values were used to compare the changes of neurological scores. Data of MWM were analyzed by one-way ANOVA nonparametric test of kruskal-wallis. Differences between more three groups were analyzed using one-way ANOVA following Tukey's test. Differences between two groups were compared by t tests. A probability of value of $P<0.05$ was considered to be statistically significant.

The other materials and methods used in this study are described in the Supplemental material.

\section{Results}

\section{General Observations and Mortality Rate of Rats Following SAH}

In our experiment, data regarding body weight, body temperature, arterial blood gas, and mean arterial blood pressure were measured. No significant differences between each experimental group were observed (data not shown). No rats died in the sham group both in the experiment 1 and 2 . The mortality rate of SAH rats was $28 \%(42 / 150)$ in experiment 1 and $21 \%(32 / 152)$ in experiment 2, and then survival rats were used for following experiment and no died until end. As shown in Fig. $1 \mathrm{~b}$, blood clots were located at the surface of brainstem and Willis cycle, suggesting the successful establishment of SAH model in rats.

\section{Apelin-13 Alleviated Neurological Deficits and Brain Edema, Improved Spatial Memory, and Inhibited Inflammation in SAH rats}

First, we accessed the effect of apelin-13 at three concentration on neurological score and brain edema in $\mathrm{SAH}$ rats. Compared to the rats in the sham group, neurological scores and brain edema were elevated in $\mathrm{SAH}$ rats. Administration of apelin-13 at $25 \mu \mathrm{g} / \mathrm{kg}$ had no significant effects on neurological score but significantly reduced brain edema. However, the neurological scores and brain edema in apelin-13 at 50 $\mu \mathrm{g} / \mathrm{kg}$ and $100 \mu \mathrm{g} / \mathrm{kg}$ groups were significantly lower than vehicle group $(P<0.05$, Fig. 2a and 2b). Next, we evaluated the role of apelin-13 on the spatial memory of SAH rats using MWM test. As a result, swimming distance and escape latency trends were similar to the neurological score in each group, apelin-13 at $50 \mu \mathrm{g} / \mathrm{kg}$ and $100 \mu \mathrm{g} / \mathrm{kg}$ showed significant reduction (Fig. 2c-e). Finally, we accessed the regulatory role of apelin-13 on pro-inflammatory cytokine in SAH rats. The levels of IL-1 $\beta$ and TNF-a were increased after SAH induction, and apelin-13 at three concentration were suppressed the levels of IL-1 $\beta$ and TNF- $a$ in CSF samples, compared to the rats in the vehicle group (Fig. $2 \mathrm{f}$ and $1 \mathrm{~g}$ ). Moreover, the levels of IL-1 $\beta$ and TNF-a showed no difference between apelin-13 at $100 \mu \mathrm{g} / \mathrm{kg}$ and $50 \mu \mathrm{g} / \mathrm{kg}(P>0.05$, Fig. $2 f$ 
and 1g). Taken together, apelin-13 could alleviated the neurological deficit and brain edema, improve spatial memory and inhibit inflammatory response in SAH rats, and apelin-13 at the dosage of $50 \mu \mathrm{g} / \mathrm{kg}$ exhibited the most beneficial neuroprotective effect.

\section{Apelin-13 Alleviated Brain Injury Depending on APJ in SAH rats}

Given the results in Fig. 2, $50 \mu \mathrm{g} / \mathrm{kg}$ of apelin-13 was selected for subsequent rat experiments. As expected, $50 \mu \mathrm{g} / \mathrm{kg}$ of apelin-13 treatment reduced neurological scores and brain edema, improved spatial memory and inhibited the level of IL-1 $\beta$ and TNF-a in SAH rats (Fig. 3a-g). However, administration with ML221, an inhibitor of APJ, significantly weakened the above neuroprotective role of apelin-13 in SAH rats (Fig. 3a-g). Besides, the expression of APJ was significantly increased in SAH rats, and apelin-13 treatment further elevated APJ expression, but ML221 attenuated the expression of APJ and blocked the effect of apelin-13 (Fig. 4a). These results suggested that apelin-13 alleviated brain injury depended on APJ. Study reported that apelin protect dopaminergic neuron through APJ and apoptosis pathway [16]. Thus, we next investigated the effect of apelin-13/APJ on the expression of apoptosis-related proteins. As a result, the protein levels of Bax and active caspase 3 were significantly elevated while the Bcl-2 was reduced after SAH induction, compared to the sham group $(P<0.01$, Fig. 4a). Apelin-13 suppressed the expression levels of Bax and active caspase 3, but increased Bcl-2 expression in SAH rats, which suggesting apelin-13 has a protective against apoptosis in SAH rats $(P<0.01$, Fig. 4a). Whereas, ML221 significantly weakened the apoptosis inhibition of apelin-13 in SAH rats $(P<0.05$, Fig. 4a). The results of TUNEL staining also showed that apelin-13 significantly reduced the proportion of apoptotic cells, but ML221 abolished this effect (Fig. 4b). Therefore, apelin-13 inhibited neuronal apoptosis, which is dependent on APJ. Consistently, the results of FJB and Nissl staining once again confirmed the protective role of apelin-13/APJ system on neuron in SAH rats (Fig. 4c and 4d). Taken together, these findings evidenced that apelin-13 alleviated brain injury depending on APJ in SAH rats.

\section{Apelin-13 inhibited apoptosis and inflammation in an APJ-dependent manner after SAH in vitro}

To further explore the mechanism of the neuroprotective effect of apelin/APJ system, we investigated the effects of apelin/APJ system on apoptosis-related proteins and inflammatory cytokine in vitro. As shown in Supplemental Fig. 1, in neuron-microglia co-culture system, neurons showed evident neuronal degeneration characterized by swollen bodies with loss of synapses, but apelin-13 adminstration significantly prevented these morphological changes. Besides, compared with SAH + DMSO group, apelin-13 treatment significantly elevated the viability of neuron cells and the effect was most significant at $5 \mathrm{nM}$ (Supplemental Fig. 2). Thus, $5 \mathrm{nM}$ apelin-13 was used for subsequent cell experiments.

Moreover, $\mathrm{OxyHb}$ exposure led to a significant increase in APJ protein expression, and treatment with apelin-13 further enhanced APJ expression, but ML221 blocked this effect of apelin-13 on APJ (Fig $5 a)$. As expected, $0 x y H b$ exposure activated the apoptotic pathways in neurons, showed as an increase expression of Bax and Caspase-3, and decrease Bcl-2 expression (Fig 5a). Apelin-13 treatment rescued the damage of $\mathrm{OxyHb}$ to neurons, while ML221 significantly prevented the inhibitory effect of apelin- 
13 on apoptosis (Fig 5a). TUNEL results supported that apelin-13 protect neuron in an APJ dependent manner (Fig 5b). In addition, the results of western blot and IF showed that, apelin-13 promoted the levels of anti-inflammatory cotykines (IL-4 and IL-10) and inhibited the levels of pro-inflammatory cotykines (IL$1 \beta$ and TNF- $\alpha$ ) in BV-2 cells, suggesting that apelin-13 regulated inflammatory pathways after SAH in neuron (Fig $5 \mathrm{c}$ and $5 \mathrm{~d}$ ). The ML221 also could block the effect of apelin-13 on inflammation regulation (Fig 5c and 5d). Collectively, apelin-13 inhibited apoptosis and inflammation in an APJ-dependent manner after SAH in vitro.

\section{Discussion}

Just like a cell signal system, apelin binding APJ to active second messenger signaling cascades [17]. However, up to now, the relationship between apelin/APJ system and SAH has not been clarified clearly. In our study, we demonstrated that apelin-13 exhibit a neuroprotective role through alleviating neurological deficits, promoting spatial memory, and inhibiting inflammation in SAH rats, thereby improve EBI. Mechanically, apelin-13 inhibited apoptosis signal and inflammatory cytokine (TNF-a and IL-1ß) in an APJ dependent manner (Fig. 5e).

Xu et al. found that apelin-13 could alleviate the EBI following SAH by suppressing ER stress-mediated apoptosis possibly involving Activating transcription factor 6/CCAAT/enhancer-binding protein homologous protein (ATF6/CHOP) pathway [18]. Another study by Liu et al. revealed that the neuroprotective role of apelin-13 is mediated by GLP-1R/PI3K/Akt pathway [19]. However, those studies had some limitations and did not fully reveal the mechanisms involved in EBI and they also did not evaluate the relationship between APJ and apelin-13. Whereas, the apelin-13/APJ system is important in the whole pathological process and effects the outcome of SAH. The data from Xu et al also indicated apelin-13 binding to APJ can attenuate early brain injury by reducing ER stress-mediated oxidative stress and neuroinflammation [20]. In our experiment, to further evidence the role of the apelin-13/APJ system in SAH-induced EBI, ML221, an inhibitor of APJ, was applied in combination apelin-13. As a result, the apelin-13 remarkably accelerated the expression of APJ while ML221 (an inhibitor of APJ) blocked this effect, which precisely illustrated the dependence between apelin-13 and APJ. Thus, we concluded that apelin-13 protect against brain damage after SAH in an APJ dependent manner. It is worth mentioning that the antagonistic effect of ML221 on APJ appears to be an apelin-dependent. For example, Roche et al reported that ML221 did not indicate significant effect on progesterone production or proliferation in bovine ovarian cells unless cells were stimulated with recombinant apelin-13 [21]. Therefore, apelin-13 protect against brain damage after SAH in an APJ dependent manner. APJ mediates apelin-13 to protect against brain injury after SAH, and antagonism of ML221 to APJ may be depend on apelin-13.

Apelin-13 can regulate the apoptotic signaling pathway and thus reduce the number of apoptotic neurons in the cortex during $\mathrm{SAH}$, which may be another mechanism of apelin-13 to protect brain tissue. Apoptosis is a kind of program cell death pathway and play a crucial role in EBI induced by SAH. Proapoptotic Bcl-2 family members take part in and activate caspase- 9 to trigger further the downstream effector caspases, including caspase-3, caspase-6, and caspase-7 through the intrinsic apoptotic 
pathway [22]. As is well known, cell apoptosis is enhanced after SAH, where Bax and caspase 3 were upregulated, while Bcl-2, an apoptotic inhibitor, was down-regulated. Importantly, previous study reported that apelin shows strong anti-apoptosis effect and ameliorates EBI after SAH in rats [19]. Another study showed that apelin-13 pre-treatment can diminish memory impairment by inhibiting apoptosis in an Alzheimer's disease [23, 24]. In our study, the cell apoptosis was reflected by apoptosis-related markers, the expression levels of Bax and active caspase 3 were significantly elevated while Bcl-2 was suppressed in rats after SAH, but apelin-13 partially revised these effect. Furthermore, ML221 could partially reduce the effect of apelin-13 on apoptosis-related marker. Thus, apelin-13 could protect against cell apoptosis after SAH via intervening the expression of $\mathrm{Bax}, \mathrm{Bcl}-2$ and active caspase 3 in an APJ dependent manner. In addition, TUNEL, FJB and Nissl staining also indicated that apelin-13 decreased the cell death and attenuated SAH-induced EBI in an APJ dependent manner. Taken together, these results revealed that apelin-13 exhibits neuroprotective role against SAH-induced EBI through attenuating apoptosis.

We also realize there were some limitations in our work. To be first, the present study only explored the neuroprotective effects of apelin-13 in the early stages of SAH to male rats. Thus, its long-term neuroprotective roles and sex difference require further investigation in SAH-induced brain damage and neurological deficits. Secondly, our study demonstrated that apelin-13 can only partially attenuate neuronal apoptosis dependent on APJ expression, implying other molecular mechanisms involved which need further investigation.

\section{Conclusion}

In summary, our current study confirms that apelin-13 exhibits the neuroprotective role to attenuate EBI following $\mathrm{SAH}$, which is mediated by inhibiting neuronal apoptosis in an APJ dependent manner following SAH in rats. These findings confirmed that apelin-13 could be developed as a novel drug for treatment of SAH. Apelin-13/APJ system as an endogenous signaling pathway played a crucial role during $\mathrm{SAH}$ and should be explored further in the future.

\section{Abbreviations}

ANOVA Analysis of variance

APJ G protein-coupled receptor-apelin and angiotensin-1-like receptor

Bax $\quad$ Bcl-2 associated $x$ protein

Bcl-2 B-cell lymphoma 2

CSF Cerebral spinal fluid

DAPI 4',6-diamidino-2-phenylindole

DMSO Dimethylsulfoxide 
dUTP 2'-Deoxyuridine 5'-Triphosphate

EBI Early brain injury

ER Endoplasmic reticulum

FJB Fluoro-Jade B

GLP-1R/PI3K/Akt Glucagon-like peptide-1/phosphatidylinositol 3-kinase/protein kinase B

IL-1 $\beta \quad$ Interleukin-1 $\beta$

PBS phosphate buffer solution

ML221 4-oxo-6-((pyrimidin-2-ylthio)methyl)-4H-pyran-3-yl 4-nitrobenzoate

MWM Morris water maze

Nrf2 Nuclear factor erythroid 2-related factor-2

ROS Reactive oxide species

SAH Subarachnoid hemorrhage

SD Sprague-Dawley

TNF-a Tumor necrosis factor-a

WW/DW wet weight/ dry weight

Means \pm SD means \pm standard deviation

IF immunofluorescence

MTT 3-(4,5-Dimethylthiazol-2-yl)-2,5-diphenyltetrazolium bromide

\section{Declarations}

\section{Funding}

This work was supported by the National Natural Science Foundation of China (No. 81873741 \& No.82071297).

\section{Conflicts of interest}

The authors declare that they have no competing interest. 


\section{Authors' contributions}

All authors had full access to all the data in the study and take responsibility for the integrity of the data and the accuracy of the data analysis. Study concept and design: Gang Chen, Zhong Wang and Haitao Shen. Acquisition of data: Xiaoyan Shen, and Guiqiang Yuan. Analysis and interpretation of data: Bing Li, Cheng Cao, Demao Cao, and Jiang Wu. Drafting manuscript: Xiaoyan Shen, and Guiqiang Yuan. Administrative, technical, and material support: Haiying Li, and Xiang Li. Study supervision: Gang Chen, Zhong Wang and Haitao Shen.

\section{Ethics approval}

All animal experiments were approved by the Institutional Animal Care Committee of Soochow University and were performed in accordance with the guidelines of the National Institutes of Health.

\section{Acknowledgments}

The authors thank Mr. Zhi Li for his linguistic assistance during the preparation of this manuscript.

Apelin-13 attenuates early brain injury through inhibiting inflammation and apoptosis in rats after experimental subarachnoid hemorrhage

\section{References}

1. Macdonald RL and Schweizer TA (2017) Spontaneous subarachnoid haemorrhage. Lancet 389: 655666. doi: 10.1016/S0140-6736(16)30668-7

2. Balbi M, Vega MJ, Lourbopoulos A et al. (2019) Long-term impairment of neurovascular coupling following experimental subarachnoid hemorrhage. J Cereb Blood Flow Metab: 271678X19863021. doi: $10.1177 / 0271678 \times 19863021$

3. Suzuki H (2019) Inflammation: a Good Research Target to Improve Outcomes of Poor-Grade Subarachnoid Hemorrhage. Transl Stroke Res 10: 597-600. doi: 10.1007/s12975-019-00713-y

4. Mejdoubi M, Schertz M, Zanolla S et al. (2018) Transoceanic Management and Treatment of Aneurysmal Subarachnoid Hemorrhage: A 10-Year Experience. Stroke 49: 127-132. doi: 10.1161/STROKEAHA.117.017436

5. Xie YK, Zhou X, Yuan HT et al. (2019) Resveratrol reduces brain injury after subarachnoid hemorrhage by inhibiting oxidative stress and endoplasmic reticulum stress. Neural Regen Res 14: 1734-1742. doi: 10.4103/1673-5374.257529

6. Conzen C, Becker K, Albanna W et al. (2019) The Acute Phase of Experimental Subarachnoid Hemorrhage: Intracranial Pressure Dynamics and Their Effect on Cerebral Blood Flow and Autoregulation. Transl Stroke Res 10: 566-582. doi: 10.1007/s12975-018-0674-3

7. Naraoka M, Fumoto T, Li Y et al. (2019) The Role of Intracranial Pressure and Subarachnoid Blood Clots in Early Brain Injury After Experimental Subarachnoid Hemorrhage in Rats. World Neurosurg 
129: e63-e72. doi: 10.1016/j.wneu.2019.05.009

8. Kleinz MJ and Davenport AP (2005) Emerging roles of apelin in biology and medicine. Pharmacol Ther 107: 198-211. doi: 10.1016/j.pharmthera.2005.04.001

9. Bao H, Yang X, Huang Y et al. (2016) The neuroprotective effect of apelin-13 in a mouse model of intracerebral hemorrhage. Neurosci Lett 628: 219-224. doi: 10.1016/j.neulet.2016.06.046

10. Duan J, Cui J, Yang Z et al. (2019) Neuroprotective effect of Apelin 13 on ischemic stroke by activating AMPK/GSK-3beta/Nrf2 signaling. J Neuroinflammation 16: 24. doi: 10.1186/s12974-0191406-7

11. Tian X, Sun L, Feng D et al. (2017) HMGB1 promotes neurovascular remodeling via Rage in the late phase of subarachnoid hemorrhage. Brain Res 1670: 135-145. doi: 10.1016/j.brainres.2017.06.001

12. Yang $Y$, Zhang $X$, Cui $\mathrm{H}$ et al. (2014) Apelin-13 protects the brain against ischemia/reperfusion injury through activating PI3K/Akt and ERK1/2 signaling pathways. Neurosci Lett 568: 44-49. doi: 10.1016/j.neulet.2014.03.037

13. Xiong Q, He W, Wang H et al. (2017) Effect of the spinal apelinAPJ system on the pathogenesis of chronic constriction injuryinduced neuropathic pain in rats. Mol Med Rep 16: 1223-1231. doi: 10.3892/mmr.2017.6734

14. Zhang Z, Wu Y, Yuan S et al. (2018) Glutathione peroxidase 4 participates in secondary brain injury through mediating ferroptosis in a rat model of intracerebral hemorrhage. Brain Res 1701: 112-125. doi: 10.1016/j.brainres.2018.09.012

15. Li P, Li X, Deng P et al. (2020) Activation of adenosine A3 receptor reduces early brain injury by alleviating neuroinflammation after subarachnoid hemorrhage in elderly rats. Aging (Albany NY) 13 : 694-713. doi: 10.18632/aging.202178

16. Angelopoulou E, Paudel YN, Bougea A et al. (2021) Impact of the apelin/APJ axis in the pathogenesis of Parkinson's disease with therapeutic potential. J Neurosci Res. doi: 10.1002/jnr.24895

17. O'Donnell LA, Agrawal A, Sabnekar P et al. (2007) Apelin, an endogenous neuronal peptide, protects hippocampal neurons against excitotoxic injury. J Neurochem 102: 1905-1917. doi: 10.1111/j.14714159.2007.04645.x

18. Xu W, Gao L, Li T et al. (2018) Apelin-13 Alleviates Early Brain Injury after Subarachnoid Hemorrhage via Suppression of Endoplasmic Reticulum Stress-mediated Apoptosis and Blood-Brain Barrier Disruption: Possible Involvement of ATF6/CHOP Pathway. Neuroscience 388: 284-296. doi: 10.1016/j.neuroscience.2018.07.023

19. Liu Y, Zhang T, Wang Y et al. (2019) Apelin-13 attenuates early brain injury following subarachnoid hemorrhage via suppressing neuronal apoptosis through the GLP-1R/PI3K/Akt signaling. Biochem Biophys Res Commun 513: 105-111. doi: 10.1016/j.bbrc.2019.03.151

20. Xu W, Li T, Gao L et al. (2019) Apelin-13/APJ system attenuates early brain injury via suppression of endoplasmic reticulum stress-associated TXNIP/NLRP3 inflammasome activation and oxidative stress in a AMPK-dependent manner after subarachnoid hemorrhage in rats. J Neuroinflammation 16: 247. doi: 10.1186/s12974-019-1620-3

Page $13 / 20$ 
21. Roche J, Rame C, Reverchon M et al. (2017) Apelin (APLN) regulates progesterone secretion and oocyte maturation in bovine ovarian cells. Reproduction 153: 589-603. doi: 10.1530/REP-16-0677

22. Datta A, Sarmah D, Mounica L et al. (2020) Cell Death Pathways in Ischemic Stroke and Targeted Pharmacotherapy. Transl Stroke Res. doi: 10.1007/s12975-020-00806-z

23. Aminyavari S, Zahmatkesh M, Farahmandfar M et al. (2019) Protective role of Apelin-13 on amyloid beta25-35-induced memory deficit; Involvement of autophagy and apoptosis process. Prog Neuropsychopharmacol Biol Psychiatry 89: 322-334. doi: 10.1016/j.pnpbp.2018.10.005

24. Zou Y, Wang B, Fu W et al. (2016) Apelin-13 Protects PC12 Cells from Corticosterone-Induced Apoptosis Through PI3K and ERKs Activation. Neurochem Res 41: 1635-1644. doi: 10.1007/s11064016-1878-0

\section{Figures}



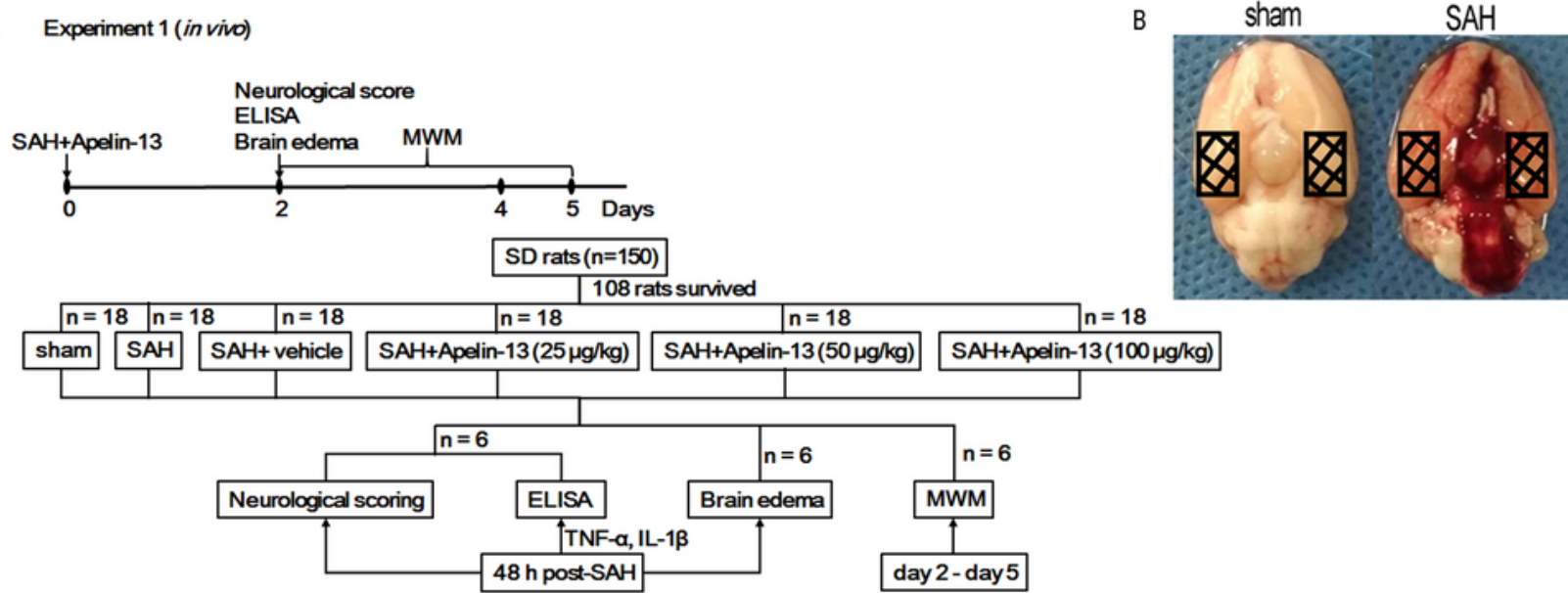

c
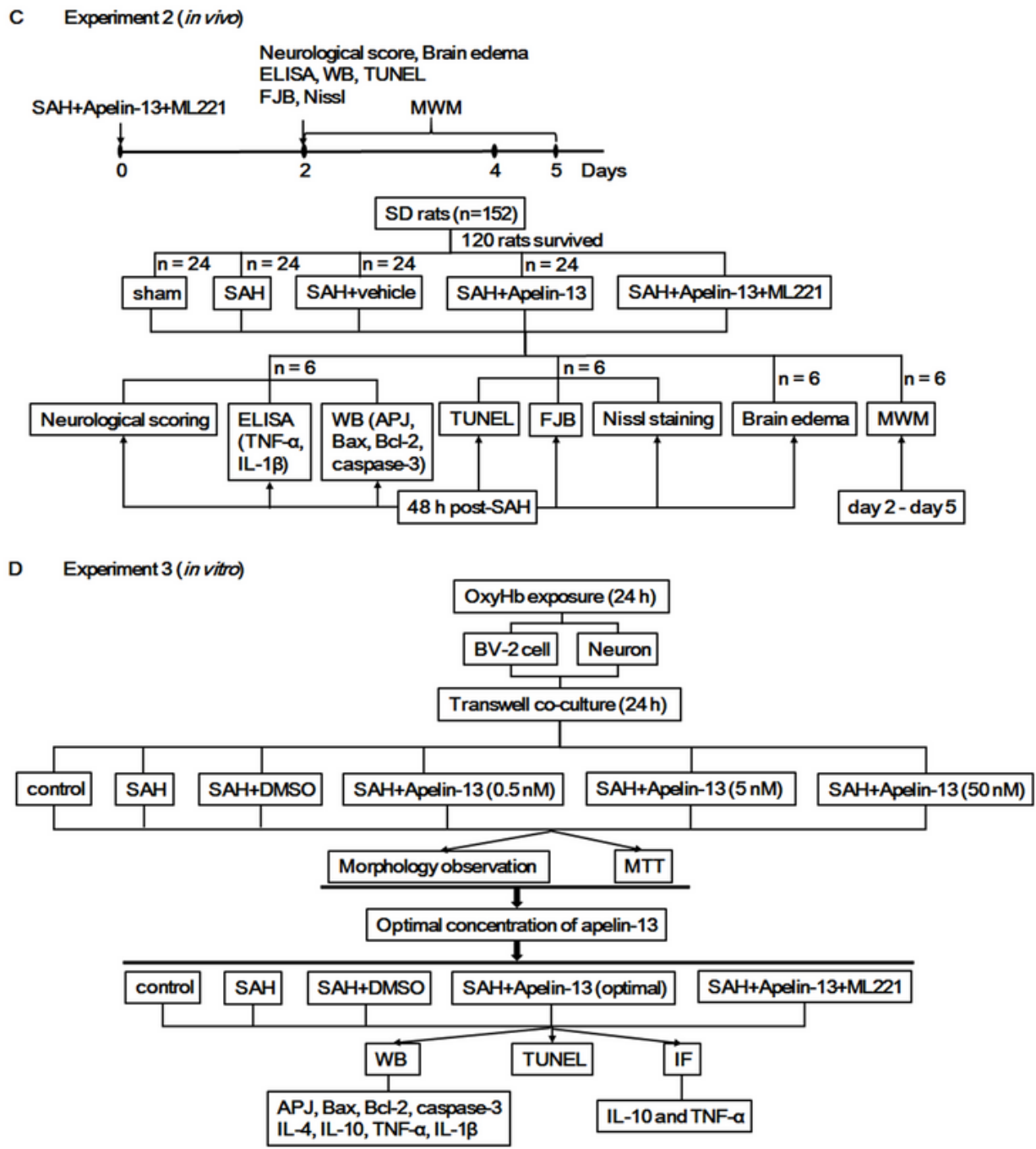

\section{Figure 1}

Establishment of SAH model in rats and study design. (a) In the Experiment 1, the timeline and groups. A total of 150 male SD rats were used and 108 rats were survived, which were then randomly divided to 6 groups ( $n=18$ ): sham group, SAH group, SAH+vehicle (saline) group, SAH+apelin-13 at $25 \mu \mathrm{g} / \mathrm{kg}, 50$ $\mu \mathrm{g} / \mathrm{kg}$ or $100 \mu \mathrm{g} / \mathrm{kg}$ groups. (b) Schematic representation of the sham group and the SAH group, the dash area on the temporal cortex showed brain tissues used for western blot analysis. (c) In the Experiment 2, 
the timeline and groups. A total of 152 male SD rats were used and 120 survived rats were randomly divided into 5 groups $(n=24)$ : sham group, SAH group, SAH+vehicle (DMSO) group, SAH+apelin-13 (50 $\mu \mathrm{g} / \mathrm{kg}$ ) group, and SAH+apelin-13+ML221 group
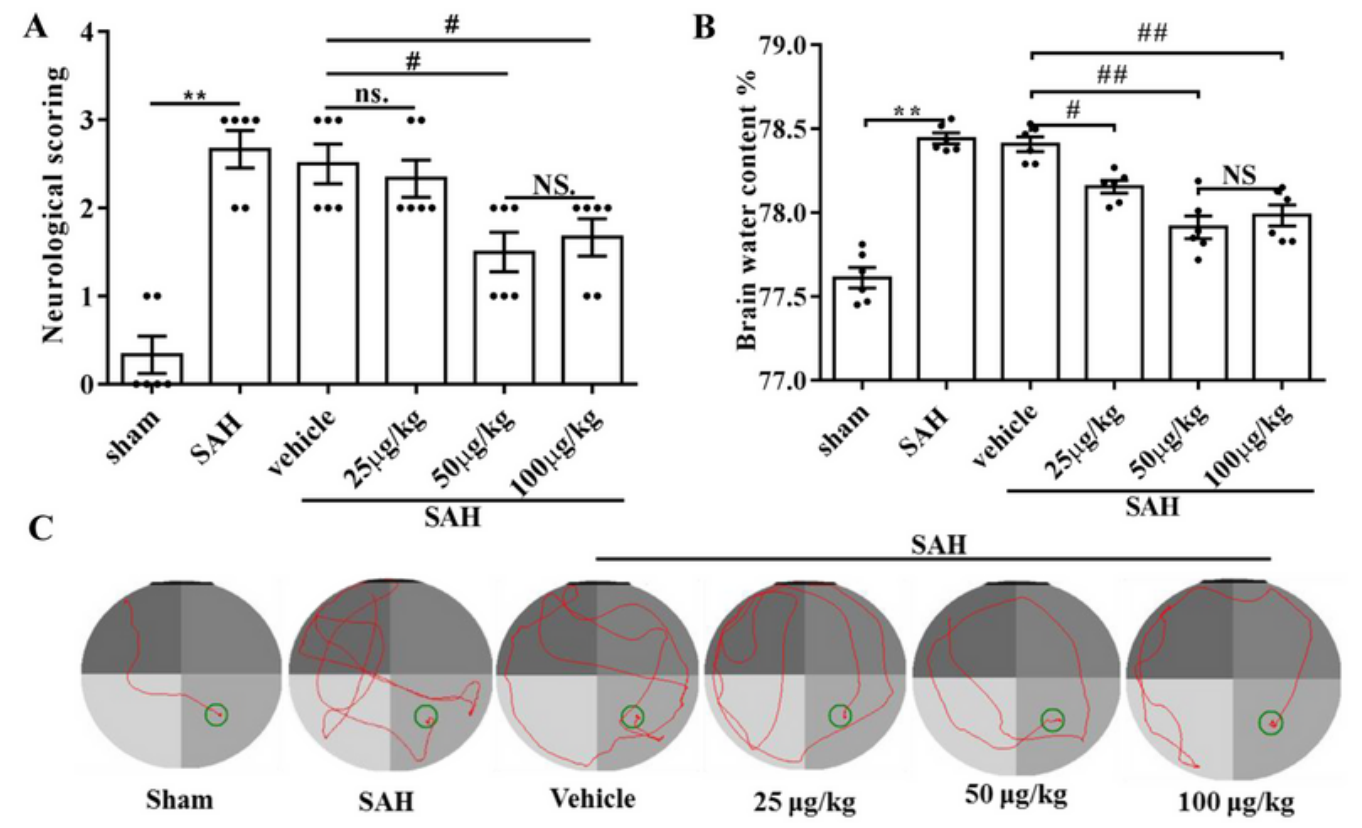

D

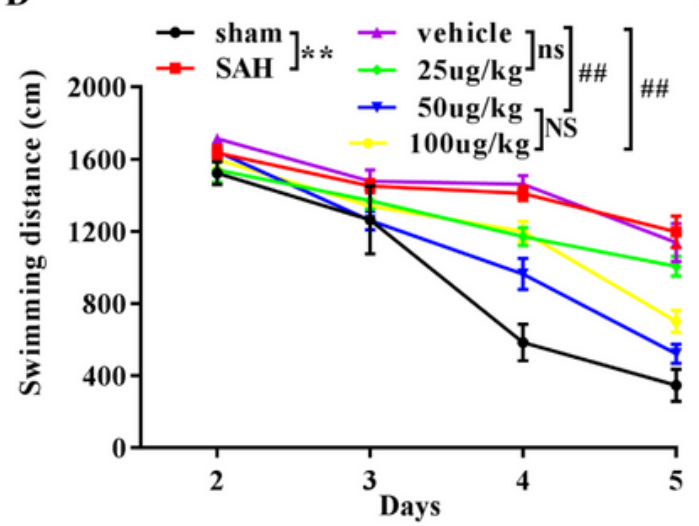

$\mathbf{F}$

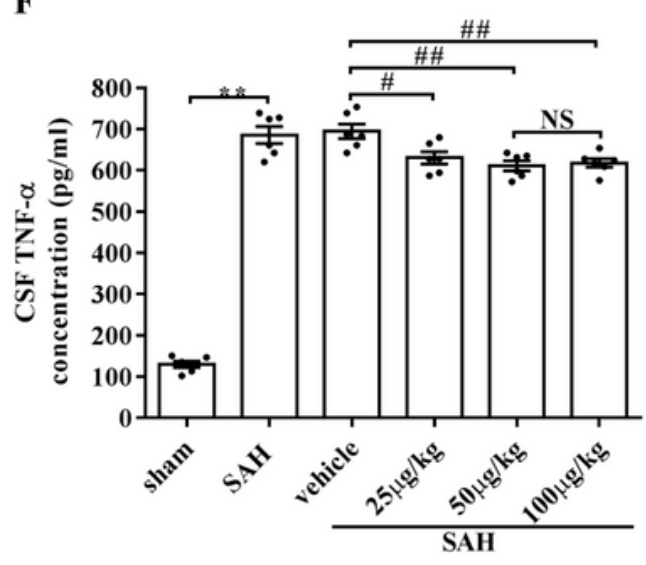

$\mathbf{E}$

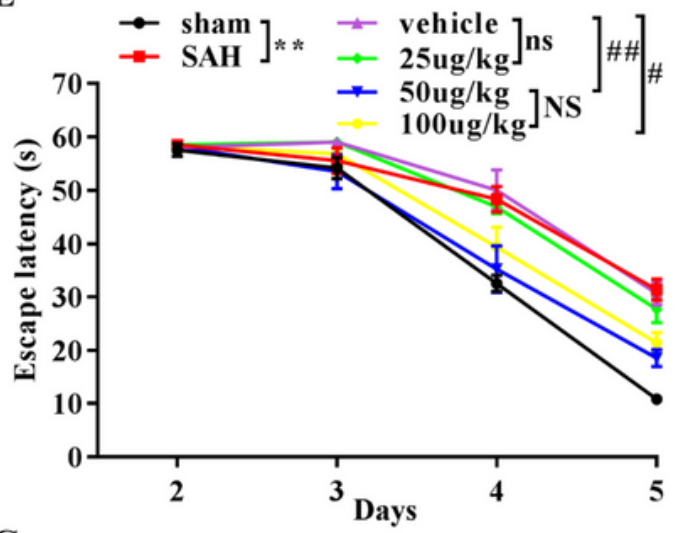

G

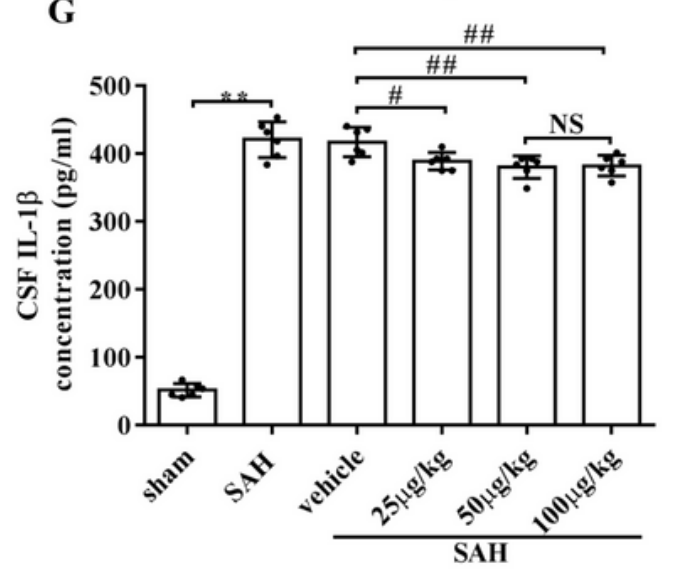

Figure 2

Apelin-13 alleviated neurological deficits, reduced brain edema, promoted spatial memory, and inhibited inflammation after SAH. (a) Compared to the sham group, SAH rats showed higher neurological scores. 
Apelin-13 at $50 \mu \mathrm{g} / \mathrm{kg}$ and $100 \mu \mathrm{g} / \mathrm{kg}$ decreased neurological scores. (b) The brain edema was determined by the percentage of brain water content. Apelin-13 treatment reduced cerebral edema induced by SAH. (c) The locus diagram of MWM. The swimming distance (d) and escape latency (e) in SAH rats treated with or without apelin-13. The TNF- $\alpha(f)$ and IL-1 $\beta(g)$ in CSF contents in SAH rats treated with or without apelin-13. All data were expressed as mean $\pm S D\left(n=6\right.$, each group). ${ }^{*} P<0.01$ vs sham group, $\# \mathrm{P}<0.05$ and \#\#P $<0.01$ vs $\mathrm{SAH}+$ vehicle group, $\mathrm{nsP}>0.05$ vs $\mathrm{SAH}+\mathrm{vehicle} \mathrm{group,} \mathrm{NSP}>0.05$ vs SAH+apelin-13 $50 \mu \mathrm{g} / \mathrm{kg}$ group
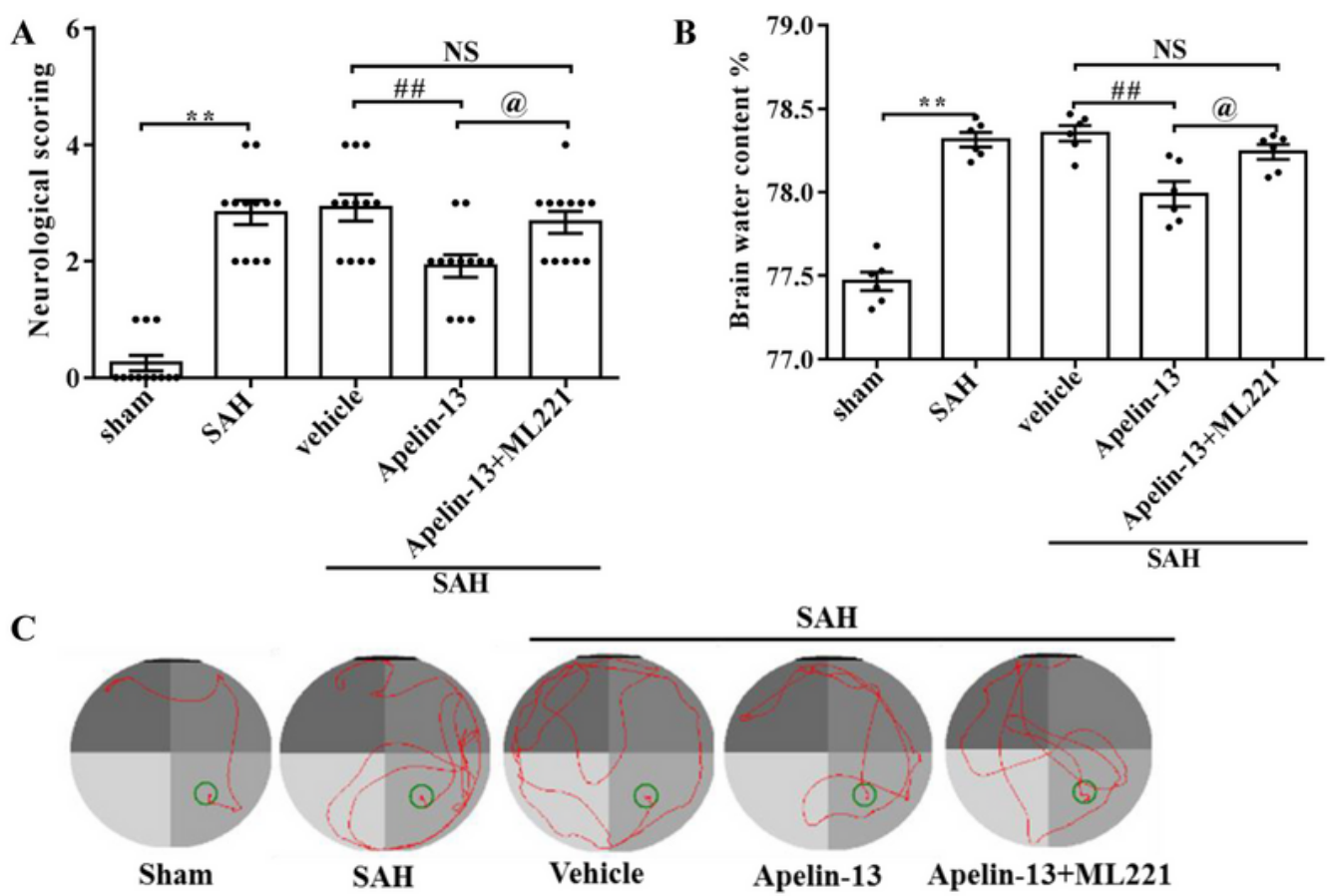

SAH
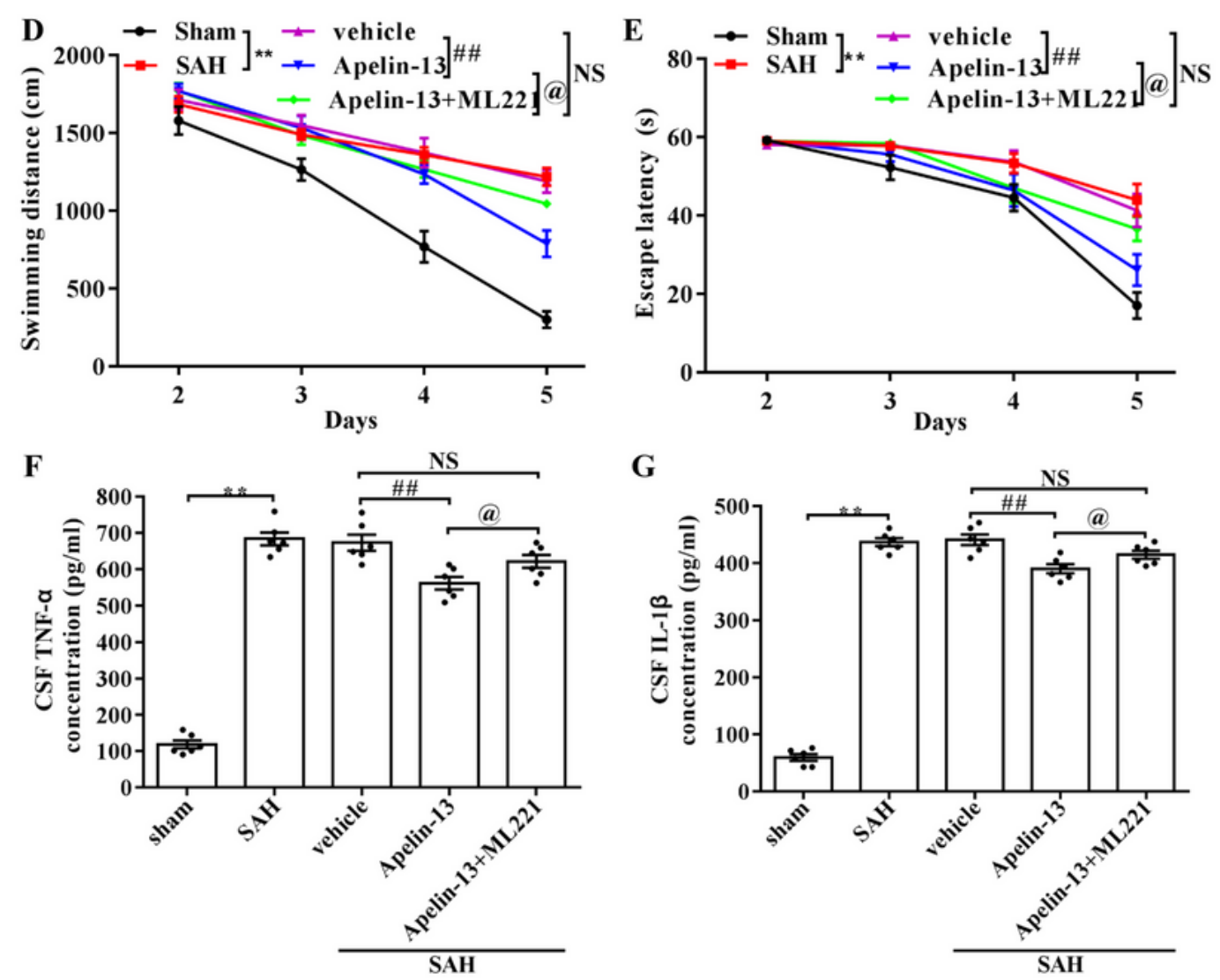


\section{Figure 3}

Apelin-13 reduced neural apoptosis after SAH dependent on APJ. (a) Apelin-13 decreased the neurological scoring. (b) Apelin-13 reduced the brain edema. (c - e) Apelin-13 decreased the swimming distance and latency time in MWM. ( $\mathrm{f}-\mathrm{g}$ ) Apelin-13 suppressed the levels of TNF- $\mathrm{a}$ and IL-1 $\beta$ in CSF. All data were represented as mean $\pm S D\left(n=6\right.$, each group). ${ }^{*} P<<0.01$ vs sham group, \#\#P<0.01 vs $S A H+v e h i c l e$ group, @P < 0.05 vs SAH+apelin-13 group, nsP®0.05 vs SAH+vehicle group
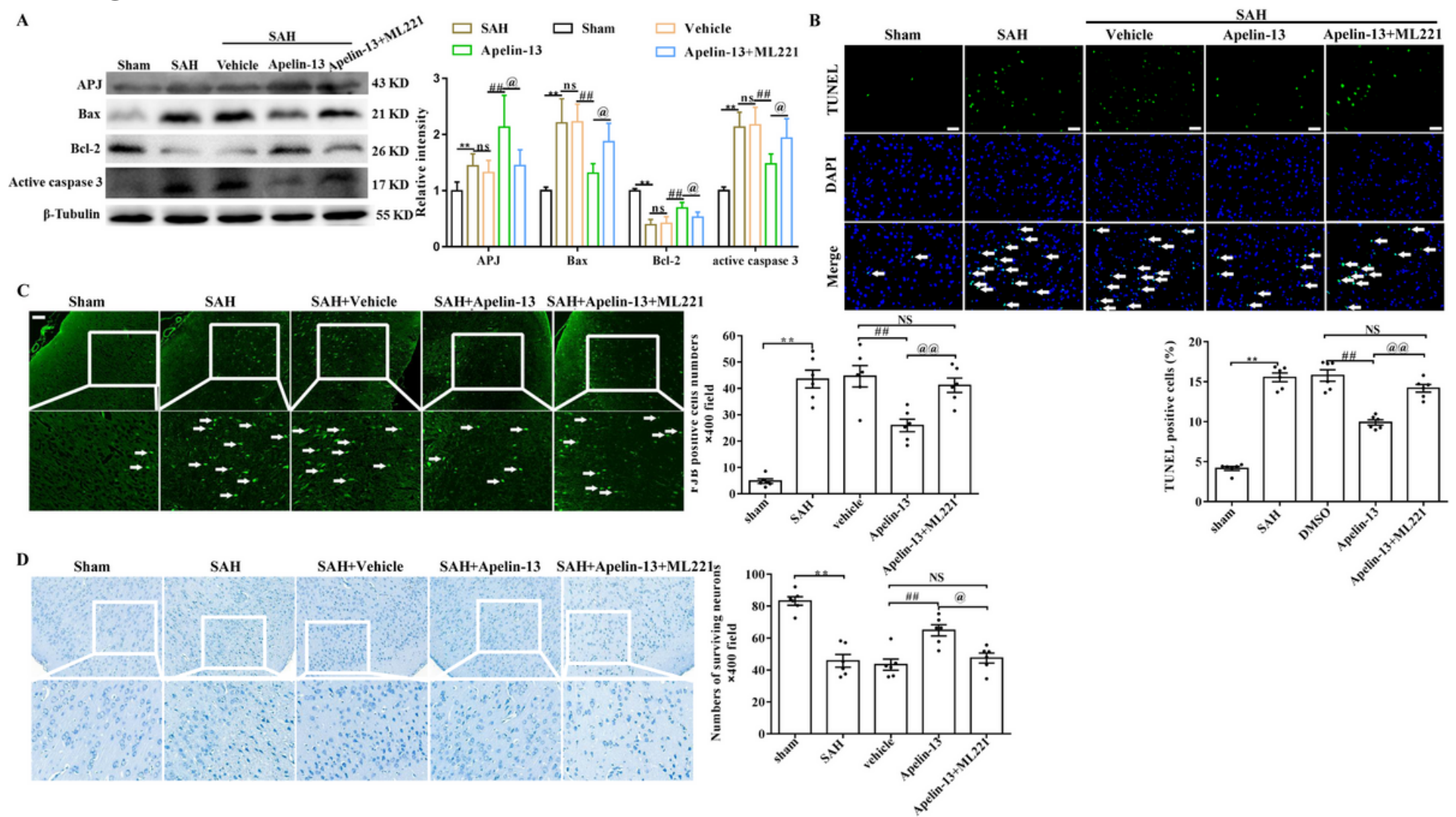

Figure 4

Apelin-13 protects against cell apoptosis and neural degeneration after SAH in an APJ dependent manner. (a) Western blot indicated the protein levels of APJ, Bax, Bcl-2, and active caspase 3 in each group. (b) Detection of apoptosis by TUNEL staining. Scale bar was $100 \mu \mathrm{m}$. Percentages of TUNEL-positive cells was also indicated. (c) FJB staining showed neuronal degeneration in the cortex of rats in different groups. Scale bar was $100 \mu \mathrm{m}$. (d) Nissl staining in the temporal cortex of rats. Quantification analysis of the number of viable neurons in temporal cortex. Scale bar was $100 \mu \mathrm{m}$. All data were described as mean $\pm S D(n=6$, each group). **P $<0.01, \# \# P<0.01$, @P $<0.05$, ns and NS $P>0.05$. 
A

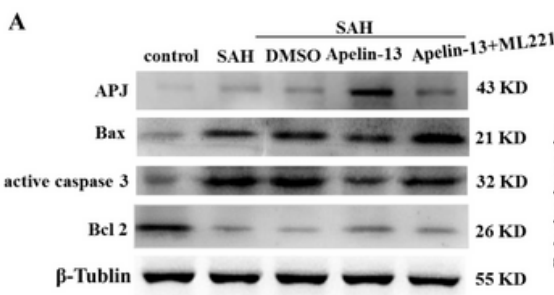

$\square$ SAH+Apelin-13
$\square$ control $\square$ SAH $\quad \square$ SAH+ Apelin-13+ML221 C
SAH DMSO

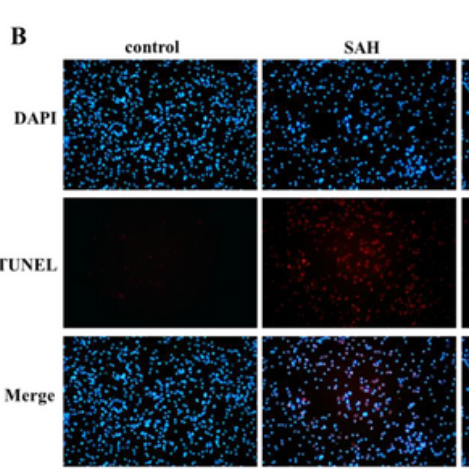

D
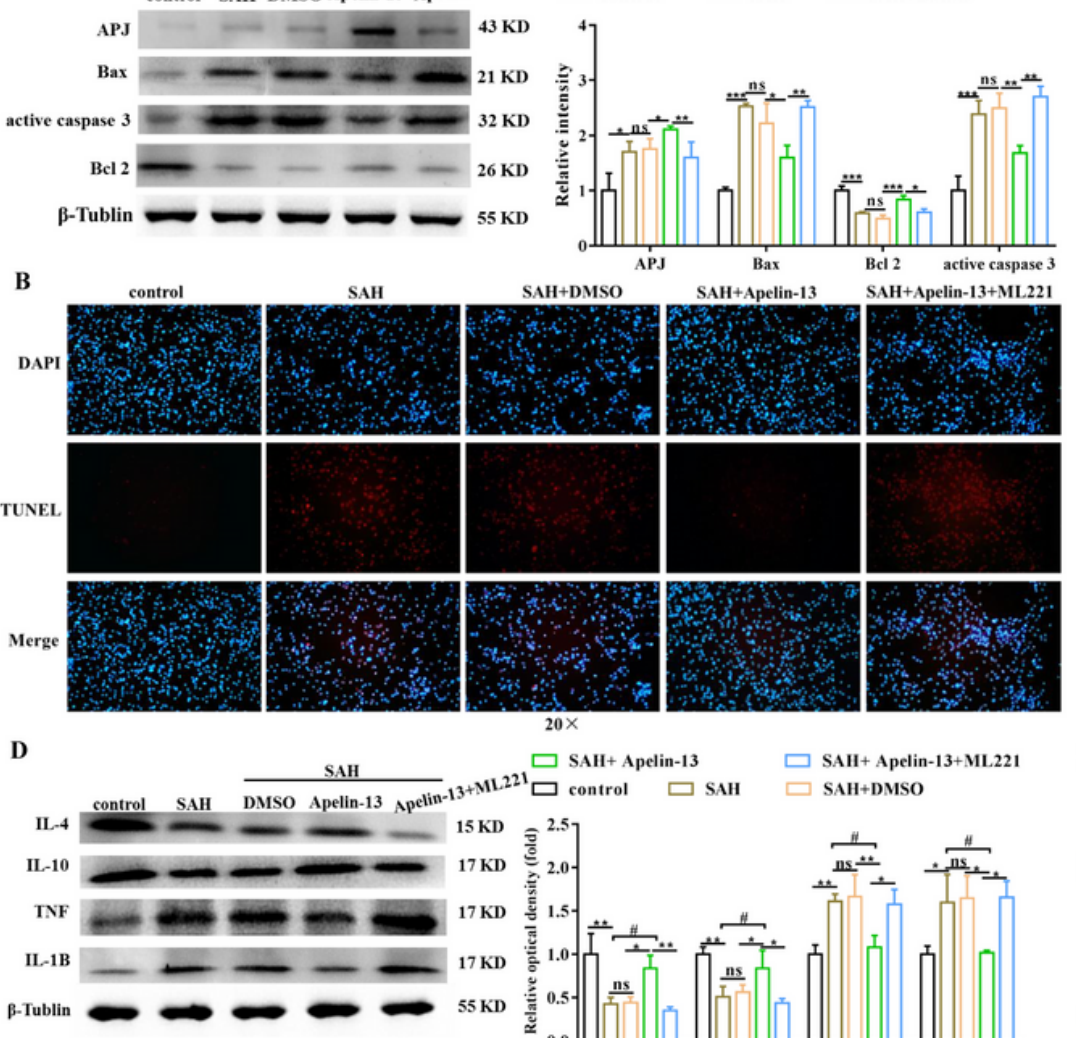
SAH+DMSO

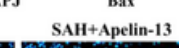

Bel 2 active caspase 3
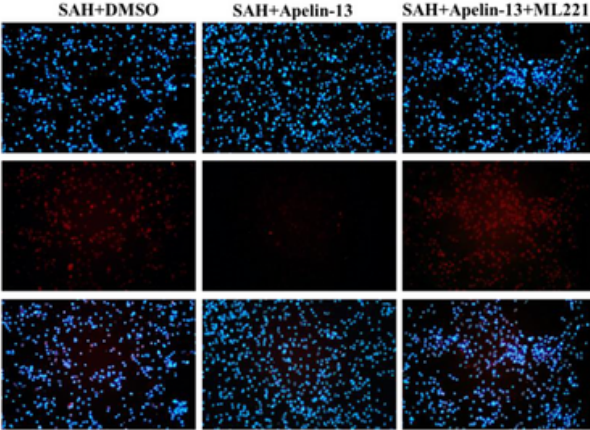

$\square \mathrm{SAH}+$ Apelin-13

$\square \mathrm{SAH}+$ Apelin-13+ML221 口 control $\square$ SAH $\square$ SAH+DMSO

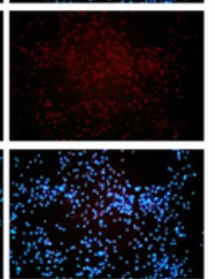

E
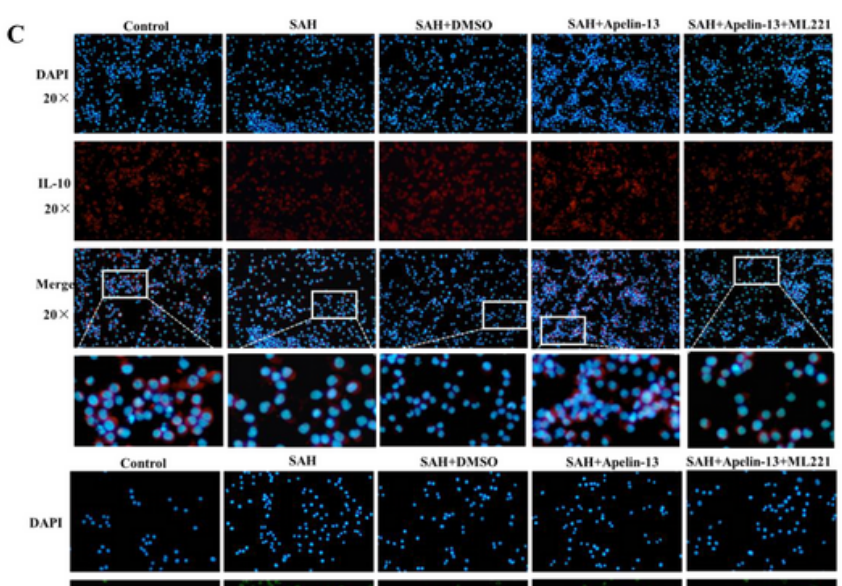

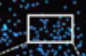

12
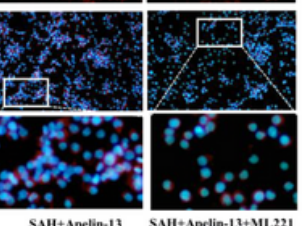

SM $\cos$
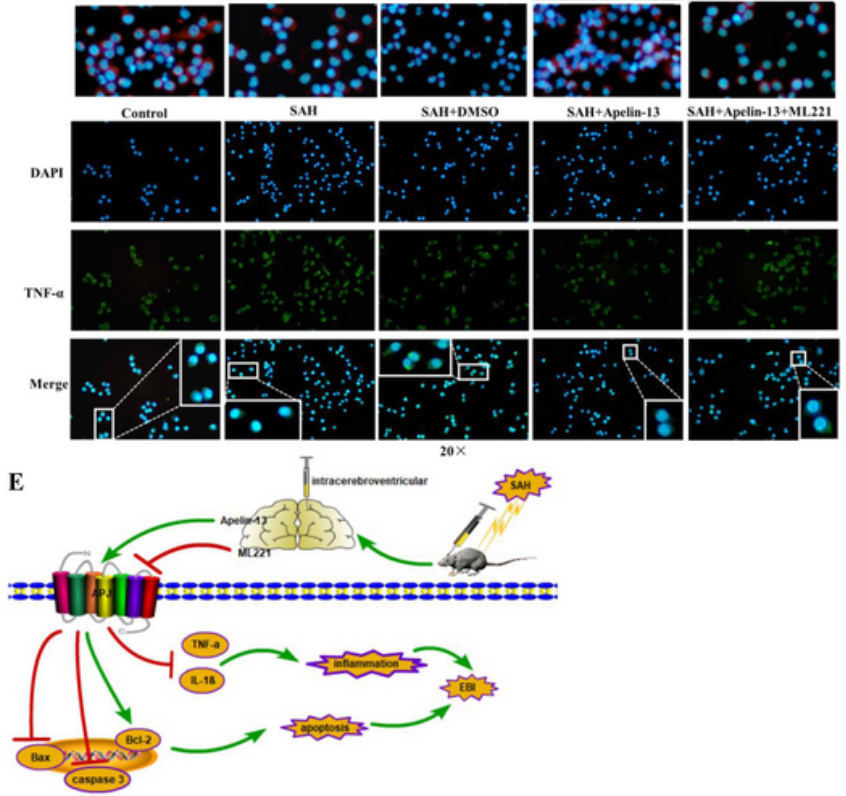

Figure 5

Apelin-13 inhibited apoptosis and inflammation in an APJ-dependent manner after SAH in vitro. (a) Western blot indicated the protein levels of APJ, Bax, Bcl-2, and active caspase 3 in each group. (b) Detection of apoptosis by TUNEL staining. Scale bar was $100 \mu \mathrm{m}$. (c) The content of TNF- $a$ and IL-10 was detected by immunofluorescence. Magnification was $20 \times$. (d) Western blot indicated the protein levels of IL-4, IL-10, TNF-a, and IL-1 $\beta$ in BV-2 cells. (e) Schematic diagram of the possible mechanism of apelin-13/APJ system on EBI after SAH. The present study illustrates that, following establishment of $\mathrm{SAH}$, the pro-apoptosis protein of Bax and active caspase 3 were significantly increased, while the antiapoptosis protein of $\mathrm{Bcl}-2$ was suppressed. The excessive cell apoptosis lead to the brain injury. On the contrary, exogenous apelin-13 suppresses the expression of pro-apoptosis proteins, and promoted Bcl-2 expression, which attenuates the EBI through inhibiting apoptosis. In addition, the neuroprotective role of apelin-13 can be blocked by ML221 via inhibiting expression of APJ receptor.

\section{Supplementary Files}

This is a list of supplementary files associated with this preprint. Click to download.

- SupplementalFig1.tif

- SupplementalFig2.tif

- SupplementalTable1.docx 
- Supplementalmaterial.docx

Page 20/20 